\title{
Probing biexciton structure in CdSe nanocrystals using 2D optical spectroscopy
}

\author{
Hélène Seiler ${ }^{1,}{ }^{*}$, Samuel Palato ${ }^{1}$, and Patanjali Kambhampati ${ }^{1}$ \\ ${ }^{1}$ Department of Chemistry, McGill University, 801 Sherbrooke St. W., H3A 0B8, Montréal, Canada
}

\begin{abstract}
Coherent Multi-dimensional Spectroscopy is ideally suited to investigate many-body effects in semiconductor nanostructures. Here we employ 2D optical spectroscopy on the model system of CdSe quantum dots to reveal the structure of the bandedge biexciton.
\end{abstract}

\section{Introduction}

A famous property of quantum confined semiconductor nanostructures is their ability to host multiple excitations. When several excitons are created within the same nanostructure, these excitons form a bound quasi-particle called a multi-exciton (MX). Despite being higher-order excitations, the physics of MXs dictates performance in potential lasing and light-emitting applications based on such nanostructures. At the fundamental level, investigating the structure and dynamics of MXs in model nanostructures is desirable as a direct experimental probe of many-body phenomena.

Coherent Multi-Dimensional Spectroscopy (CMDS) has already demonstrated its suitability to probe many-body effects in GaAs quantum wells [1-2]. Here we show how the CMDS method can similarly be applied to study exciton-exciton correlations in colloidal nanocrystals. We perform absorptive two-dimensional spectroscopy (2DE) to gain insights into the structure of the bandedge biexciton, which coarse structure is displayed in Fig. 1(a). Our data reveals that the structure of the biexciton must consist of a manifold of discrete electronic states.

\section{Results and discussion}

An example 2D spectrum obtained on an ensemble of colloidal CdSe nanocrystals is shown in Fig. 1(b), and the signals contributing to the spectrum are shown in Fig. 1(c). By spreading the optical response along two independent energy axes, effects of sample heterogeneity can be disentangled from homogeneous contributions. This key advantage of the 2DES method is exploited in our analysis. Colloidal semiconductor quantum dots feature a typical size dispersion of $5-10 \%$ owing to the wet synthesis methods by which they are produced. The size distribution of nanocrystals is responsible for crowding onedimensional spectroscopic signatures.

\footnotetext{
${ }^{*}$ Corresponding author: helene.seiler@mail.mcgill.ca
} 
(a)

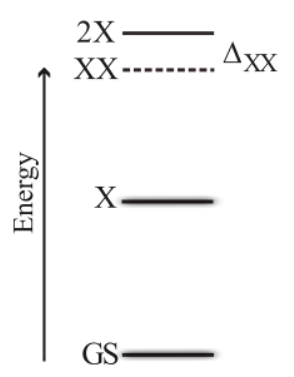

(b)
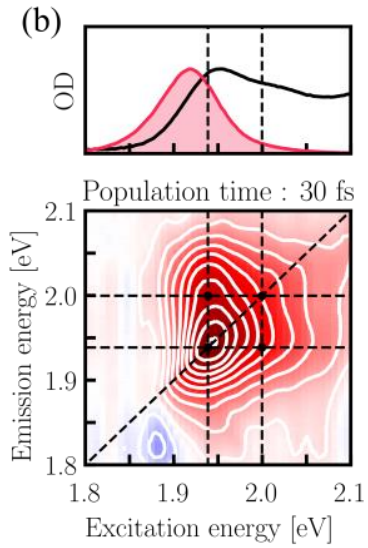

(c)

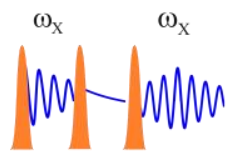

Ground State Bleach

(GSB)
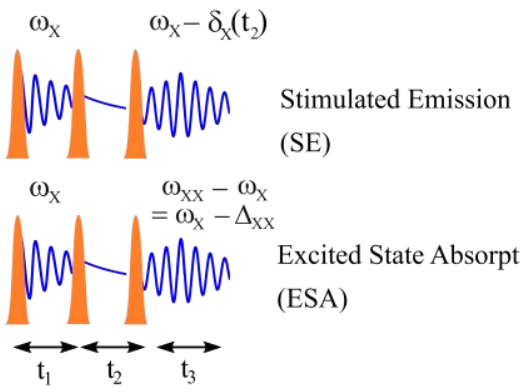

Excited State Absorption (ESA)

Fig. 1. (a) Coarse structure of the single-exciton (X) and the biexciton (XX), with a frequency detuned from $2 \mathrm{X}$ due to the binding energy $\Delta \mathrm{xx}$.(b) Example 2D correlation spectrum of colloidal CdSe nanocrystals at a population time of 30 fs. ESA shown in blue, GSB and SE in red.(c) The three signals contributing to the $2 \mathrm{D}$ spectrum. The GSB pathways oscillate at $\omega_{\mathrm{x}}$ during $\mathrm{t}_{1}$ and $\mathrm{t}_{3}$. The SE pathways oscillate at $\omega_{\mathrm{x}}$ during $\mathrm{t}_{1}$ but at $\omega_{\mathrm{x}}-\delta_{\mathrm{x}}\left(\mathrm{t}_{2}\right)$ during $\mathrm{t}_{3}$, where $\delta_{\mathrm{x}}$ is the Stokes shift. The ESA pathways oscillate at $\omega_{\mathrm{x}}$ during $\mathrm{t}_{1}$ but the frequency is detuned by the binding energy $\Delta_{\mathrm{xx}}$ during $\mathrm{t}_{3}$.

Fig. 2(a) illustrates the ability of 2DE spectroscopy to remove effects of inhomogeneity in the quantum dots sample. Spectral projections obtained by selecting slices on the 2D spectrum at various values of excitation energy are displayed. As can be seen on this panel, the bandedge peak position shifts with the excitation energy. This indicates that different sub-populations of quantum dots within the ensemble can be investigated independently.

(a)

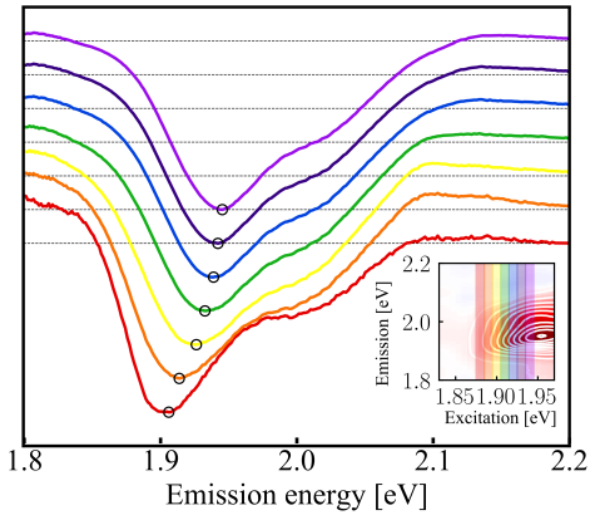

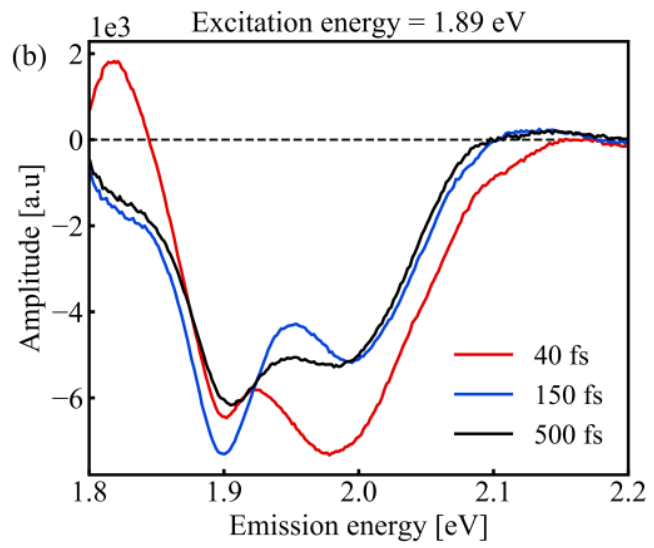

Fig. 2. (a) Pseudo-TA slices can be obtained for various values of excitation energy, here ranging from $1.88 \mathrm{eV}$ (dark red) to $1.94 \mathrm{eV}$ (purple) and for a population time of $40 \mathrm{fs}$. The colors match those shown in the inset. The minimum of each projection is indicated by a black hollow circle.(b) Projections at an excitation energy of $1.89 \mathrm{eV}$ for representative population times of $40 \mathrm{fs}$ (red), 150 fs (blue) and $500 \mathrm{fs}$ (black).

Upon selecting one of the projections shown in Fig. 2(a), one can then follow the dynamics of this sub-ensemble. Fig. 2(b) displays a series of projections at characteristic population times at an excitation energy of $1.89 \mathrm{eV}$. Each projection is a pseudo transient absorption spectrum. The most obvious change in the spectrum consists in the feature 
around $1.83 \mathrm{eV}$ which goes from positive (blue) to negative (red) values. We observe that most of the spectral changes occur within the first $100 \mathrm{fs}$ of population time, but a more subtle relaxation also takes place at later times.

The dynamics of the spectroscopic feature at $1.83 \mathrm{eV}$ reflects changes in the transition from the single exciton $\mathrm{X}$ to the biexciton state XX. Indeed biexcitonic contributions are accessed through a subset of the ESA pathways, which can be seen in Fig. 1(c). In addition to these dynamics, we also observe a blue shift of the bandedge peak as a function of population time, shown in Fig. 3 (a) and described further in [3].
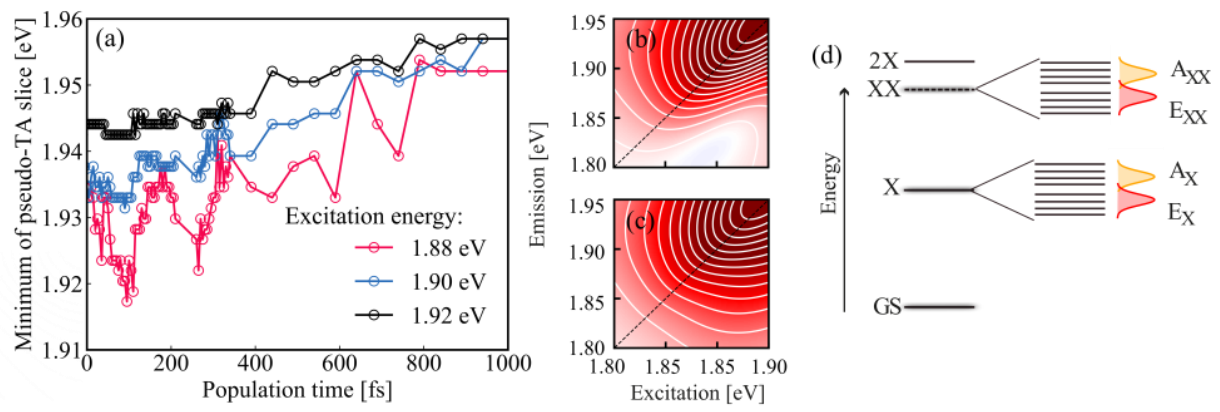

Fig. 3. (a) Minimum position of the spectral projections shown in Fig. 2.(a) as a function of population time for different excitation energies. Aside from blue shifting, the traces oscillate at the LO phonon frequency due to exciton-phonon coupling. (b) Computed 2D spectra including sample heterogeneity and multiple XX states. Parameters: inhomogeneous broadening $\sigma_{\text {inhomo }}=40 \mathrm{meV}$, homogeneous linewidth $\gamma_{\text {homo }}=30 \mathrm{meV}$, and $\Delta \mathrm{xx}=65 \mathrm{meV}$. The spectrum qualitatively matches the experimental spectra at early population times. (c) Same as in (b) but with $\Delta \mathrm{xx}=10 \mathrm{meV}$, matching the experimental spectra at later population times. (d) Updated structure for the biexciton states including a fine structure, just like within the single exciton manifold.

Using the model spectra displayed in Fig. 3. (b-c), we show that these experimental observations are consistent with population relaxation within discrete electronic states and inconsistent with a continuous energy relaxation [4]. Furthermore, our data can only be explained by invoking a manifold of discrete electronic states for the biexciton, as opposed to a single state. Fig. 3 (c) illustrates the structure of the exciton and biexciton states, as probed in the present study.

\section{Conclusion}

We have employed 2DE spectroscopy for the study of many-body effects in the model system of colloidal CdSe quantum dots. By exploiting the ability of the 2DE method to distinguish between inhomogeneous and homogeneous contributions, we have gained insights into the bandedge biexciton structure following the birth of the hot exciton.

\section{References}

1. K. W. Stone, K. Gundogdu, D.B. Turner, X. Li, S.T. Cundiff, K. Nelson, Science 80, 1169 (2009).

2. D. Karaiskaj, A.D. Bristow, L. Yang, X. Dai, R. P Mirin, S. Mukamerl, and S.T. Cundiff, Phys. Rev. Lett. 104, 1-4 (2010).

3. H. Seiler, S. Palato, P. Kambhampati, accepted in J. Chem. Phys. (2018).

4. H. Seiler, S. Palato, C. Sonnichsen, H. Baker, P. Kambhampati, Nano Lett. 18, 2999 (2018). 